\title{
Arquitecturas de sistemas de captación de energía
}

\section{(Architectures of Energy Harvesting Systems)}

\author{
Salazar Fabian¹, Núñez Maritza², Cuji Julio³, Gordón Carlos ${ }^{4}$
}

\section{Resumen}

El presente artículo desarrolla una la revisión literaria sobre las arquitecturas de sistemas de captación de energía, identificando parámetros como: frecuencia, tipo de antena, arquitectura (elementos), entre otros. La metodología tiene cuatro etapas: a) búsqueda de documentación en la que se obtuvieron 10 sistemas, los cuales fueron denominados con la letra "S" acompañada del número de artículo; b) lectura de los documentos científicos; c) extracción de información y arquitectura de los sistemas en se detallan las etapas de cada sistema (las cuales varían de 2 a 4), las frecuencias de trabajo (300 KHz hasta $3.43 \mathrm{GHz}$, siendo la de $2.45 \mathrm{GHz}$ la más empleada en sistemas para recolección de energía de radiofrecuencia). Además de utilizar, en ciertos sistemas, circuitos multiplicadores y rectificadores en diferentes configuraciones: media onda y onda completa, para posteriormente ser almacenadas en baterías o directamente aplicadas en dispositivos; d) documentación de la información extraída. Finalmente, completada la revisión literaria, se observó que en la mayoría de los artículos los sistemas tienen 3 etapas: antena, acoplamiento y rectificación, que el sistema transforma la energía recibida (corriente alterna) en corriente continua y que su funcionamiento varía en intervalos de frecuencia de 1.8 a $2.4 \mathrm{GHz}$, dependiendo de la configuración de cada sistema. Así también, el producto obtenido es una APP de consulta con un menú de selección de las diferentes arquitecturas investigadas, lo cual es un aporte muy beneficioso para los investigadores que deseen trabajar en esta área.

\section{Palabras clave}

Recolección de energía, arquitecturas, energía de radiofrecuencia, antena, acoplamiento.

\begin{abstract}
This article develops the literary review of the architectures of energy harvesting systems, identifying parameters such as: frequency, type of antenna, architecture (elements), among others. The methodology has four stages: a) Search for documentation, 10 systems were obtained, which were designated with the letter "S" accompanied by the article number; b) Reading of scientific documents; c) Extraction of information and architecture of the systems, where the stages of each system are detailed (which vary from 2 to 4), the working frequencies (300 KHz to 3.43 $\mathrm{GHz}, 2.45 \mathrm{GHz}$ being the most used in systems for collecting radio frequency energy). In addition to using in certain systems, multiplier and rectifier circuits in different configurations: half wave, full wave; to later be stored in batteries or directly applied to devices; d) Documentation of the information extracted. Finally, after completing the literary review, it was observed that, in most articles, the systems have 3 stages: antenna, coupling, and rectification that transforms the received energy (alternating current) into direct current, their operation varies in frequency intervals of 1.8 to $2.4 \mathrm{GHz}$ depending on the configuration of each system. Likewise, the product obtained is a consultation APP, with a selection menu of the different architectures investigated, which is a very beneficial contribution for researchers who wish to work in this area.
\end{abstract}

\section{Keywords}

Energy Harvesting, Architecture, Energy Radiofrequency, Antenna, Coupling.

\footnotetext{
$1 \quad$ Universidad Técnica de Ambato. Ambato, Ecuador. [fr.salazar@uta.edu.ec, https://orcid.org/0000-0002-9514-6576]

$2 \quad$ Universidad Técnica de Ambato. Ambato, Ecuador. [mnunez4777@uta.edu.ec, https://orcid.org/0000-0002-4972-0573]

3 Universidad Técnica de Ambato. Ambato, Ecuador. [julioecuji@uta.edu.ec, https://orcid.org/0000-0002-0843-735X]

4 Universidad Técnica de Ambato. Ambato, Ecuador. [cd.gordon@uta.edu.ec, https://orcid.org/0000-0002-8031-2658]
} 


\section{Introducción}

Actualmente, el término loT es muy utilizado para describir la nueva generación de interconectividad entre diferentes dispositivos, por lo cual, los sistemas de captación de energía son fundamentales en loT (Leonardo González, 2020; Dong-Hwan Park, 2014; Alok Kumar Gupta, 2019; S. Chaudhary, 2019). Su principal objetivo es eliminar el cable de alimentación, suprimir o reducir la dependencia en las baterías y mantener la vida útil de los sistemas autónomos de loT (Amit Saxena, 2019). Además, es fácil de utilizar y de bajos costes (Larrocha, 2020; Satyendra K. Vishwakarma, 2019). Ante estos requerimientos son necesarios los sistemas de recolección de energía.

Los sistemas de recolección de energía ambiental tienen diferentes arquitecturas, presentados por (Rewaa Maher, 2016: X. Bai, 2019; Hamza Tafekirt, 2020; Shanpu Shen, 2017; Hucheng Sun Y.-x. G., 2012; Robert Scheeler, 2014; Hucheng Sun Y.-x. G., 2013; Chaoyun Song, 2015; Ugur Olgun, 2010; Mahima Arrawatia, 2015; Mamta Kurvey, 2018; Ahmad A. Salih, 2016; Kyriaki Niotaki, 2013). Constan de 3 etapas básicas: antena, acoplamiento y rectificador. La antena capta las ondas de radiofrecuencia en el ambiente (Osama M. A. Dardeer, 2019) o de una fuente especifica (solar, vibraciones y movimientos) (N. A. Moghaddam, 2017). El acoplamiento asegura la transferencia de potencia entre antena y circuito rectificador (Hong, 2010) al igualar valores de impedancias de ambas etapas. Y, la rectificación convierte corriente alterna de la antena en corriente directa a su salida (Kanaya, 2014; Sun, 2020).

Otros sistemas (Richard Torrealba Meléndez, 2016) con arquitectura: antena, acoplador de impedancias, doblador y dispositivo (A.Rajan, 2014) compuestos por antena, red de adaptación, multiplicador de voltaje y almacenamiento (M Nalini J. V., 2017) están formados por: antena, convertidor RF-DC, convertidor análogo a digital, regulador de voltaje y almacenamiento (N. A. Zainuddin, 2013). Con arquitectura: antena, acoplador, rectificador, circuito de carga y batería (Anjan Kumar Kundu, 2018). Con arquitectura: antena, circuito combinador, rectificador y carga (Hadeel Aboueidah, 2017). Con arquitectura: antena, combinador, rectificador, elemento de almacenamiento, regulador de voltaje, carga (J. Martınez, 2020). E integrado por: antena, combinador de energía, convertidor RF-DC y acoplamiento incorporan una etapa adicional con el fin de obtener voltajes de salida óptimos para las necesidades del sistema. Por ello, el sistema presenta una arquitectura de 4 etapas: antena, acoplamiento, circuito multiplicador y almacenamiento. El circuito multiplicador es un tipo especial de rectificador, el cual convierte y amplifica la señal de entrada AC en una salida DC a través de la unión de rectificadores individuales en serie (Le-Giang Tran, 2017; Flandre, 2011). Normalmente, son utilizados cuando el voltaje rectificado es insuficiente para alimentar una determinada aplicación (Shailesh Singh Chouhan, 2016; Musaab Mohammed AL-Azawy, 2019). Finalmente, en la etapa de almacenamiento es en la que se acumula toda la energía recolectada por el sistema (Mohamed A. Abouzied, 2017). Usualmente, se utilizan baterías recargables y condensadores (M Nalini J. V., 2017; Yoshikawa, 2019). Otro sistema tiene una arquitectura de unificación con dos circuitos recolectores de diferentes energías (radiofrecuencia y corriente corona) (Guerrero, 2016) compuesto por: antena, circuito de recolección de corriente corona, circuito de recolección de RF, control y almacenamiento. Todos los sistemas en sus configuraciones tanto para circuitos rectificadores (Alex Mouapi, 2018; Asmaa Sedeek, 2018) como multiplicadores utilizan diodos Schottky (Halonen, 2014; H. Morales, 2012), los cuales son los más utilizados en convertidores de RF a DC (Prusayon Nintanavongsa, 2012). Sin embargo, se puede utilizar tecnología CMOS, ya que ha demostrado tener un alto porcentaje de eficiencia (P.-A. Haddad, 2014). 
El objetivo de este trabajo es hacer una revisión del estado actual de todos los sistemas de captación de energía, identificando diferentes parámetros como: frecuencia, tipo de antena, entre otros, así como la arquitectura y elementos que constituyen cada sistema.

\section{Metodología}

Se seleccionaron los artículos considerando los criterios de mejores resultados y desarrollados en la última década, los cuales fueron ordenados cronológicamente y se detallan a continuación:

- S1: Multiband Ambient RF Energy Harvesting for Autonomous loT Devices.

- S2: Radio Frequency Energy Harvesting System Making Use of $180^{\circ}$ Hybrid Couplers and Multiple Antennas to Improve the DC Output Voltage.

- S3: Sistema de cosechamiento de energía con radio frecuencia.

- S4: A High-Efficiency Broadband Rectenna for Ambient Wireless Energy Harvesting.

- S5: A Dual-Frequency Ultralow-Power Efficient 0.5-g Rectenna.

- S6: Microstrip Antenna Designs for RF Energy Harvesting.

- S7: Sistema de recolección de energía (Energy Harvesting) que emplea la corriente corona y señales de alta frecuencia.

- S8:A Compact Dual-Band Rectenna Using Slot-Loaded Dual Band Folded Dipole Antenna.

- S9: A Dual-Band Rectenna Using Broadband Yagi Antenna Array for Ambient RF Power Harvesting.

- S10: Design of a High-Efficiency 2.45-GHz Rectenna for Low-Input-Power Energy Harvesting.

Se analizó cada sistema y los datos obtenidos para cada uno son:

S1: La arquitectura de este sistema está compuesto por dos etapas. Estas son: antena y rectificador, los cuales forman una rectenna multibanda para la recolección de energía en las frecuencias de $840 \mathrm{MHz}, 1.86,2,1$ y 2,45 GHz. Las etapas del sistema se visualizan en la figura 1.

Figura 1. Diagrama de bloques del S1 y S10

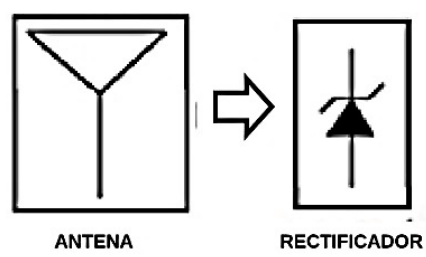

La antena es de pajarita con rendijas, el mismo que se une a un circuito rectificado de onda completa construido con rectificadores de bucle de anillo de alta eficiencia para, finalmente, ser administrada y almacenada. El sistema puede ser utilizado en aplicaciones prácticas de sensores inalámbricos (Hong Son Vu, 2020).

S2: Este sistema está compuesto por varias antenas y un acoplador hibrido de $180^{\circ}$ formando una arquitectura de 4 etapas generales que son: antenas, circuito combinador, circuito de conversión y almacenamiento. El diagrama completo se visualiza en la figura 2. 
Figura 2. Diagrama de bloques del S2

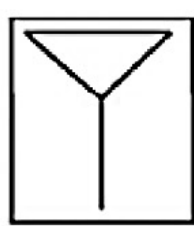

N-ANTENAS

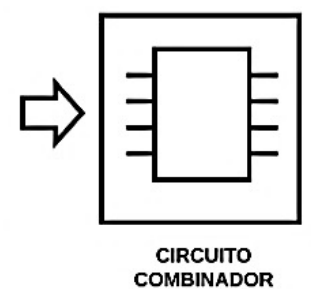

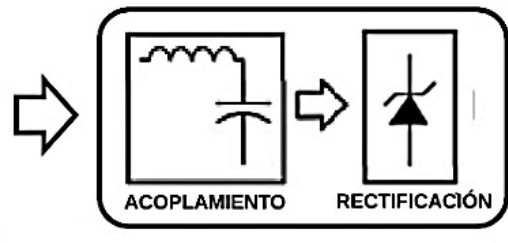

CIRCUITO DE CONVERSIÓN

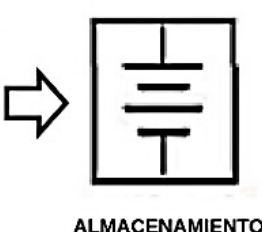

ALMACENAMIENTO

El sistema trabaja a $1.84 \mathrm{GHz}$ y recolecta energía de radiofrecuencia. La etapa de combinación y conversión tiene, en su estructura interna, un circuito multiplicador o doblador con una configuración de diodos Schottky de bajo voltaje y un rectificador de onda completa. La aplicación del sistema es en alimentación de circuitos electrónicos de bajo consumo de potencia. (J. Martınez, 2020)

S3: La arquitectura de este sistema consta de 4 etapas y es capaz de captar energía de radiofrecuencia y trabaja a una frecuencia de $915 \mathrm{MHz}$. Las etapas de este sistema se visualizan en la figura 3.

Figura 3. Diagrama de bloques del S3

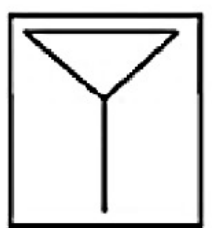

ANTENA

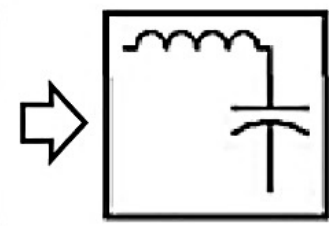

RED DE ADAPTACIÓN

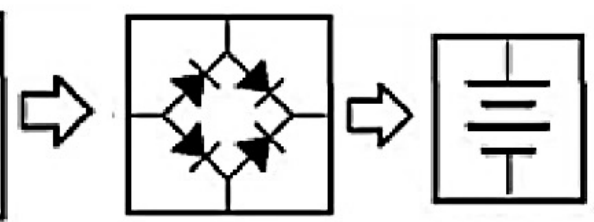

CIRCUITO MULTIPLICADOR
ALMACENAMIENTO

Las etapas del sistema son: antena, acoplador de impedancias, doblador de voltaje constituido por el circuito multiplicador y el dispositivo o almacenamiento de energía para su aplicación en circuitos de bajo consumo de potencias y recarga de baterías. La energía es recolectada con una antena monopolo espiral. Después, pasa a un rectificador de onda completa y es multiplicada por el circuito Villard (Richard Torrealba Meléndez, 2016).

S4: El sistema está diseñado para la recolección de energía inalámbrica ambiental, combinando el diseño de una antena y un rectificador acoplados. La frecuencia de trabajo en las bandas es 1.8 a $2.5 \mathrm{GHz}$. El diseño se visualiza en la figura 4 .

Figura 4. Diagrama de bloques del S4, S5 y S8

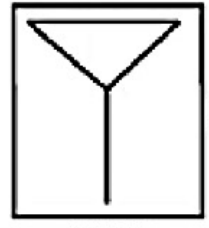

ANTENA

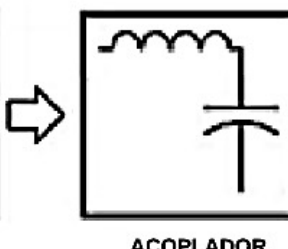

ACOPLADOR

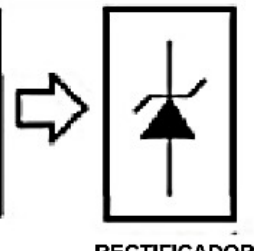

RECTIFICADOR 
El diseño de la rectena es realizado con el rectificador visualizado en la figura 5 y mostrado a continuación.

Figura 5. Diagrama de bloques del diseño del rectificador y antena para S4

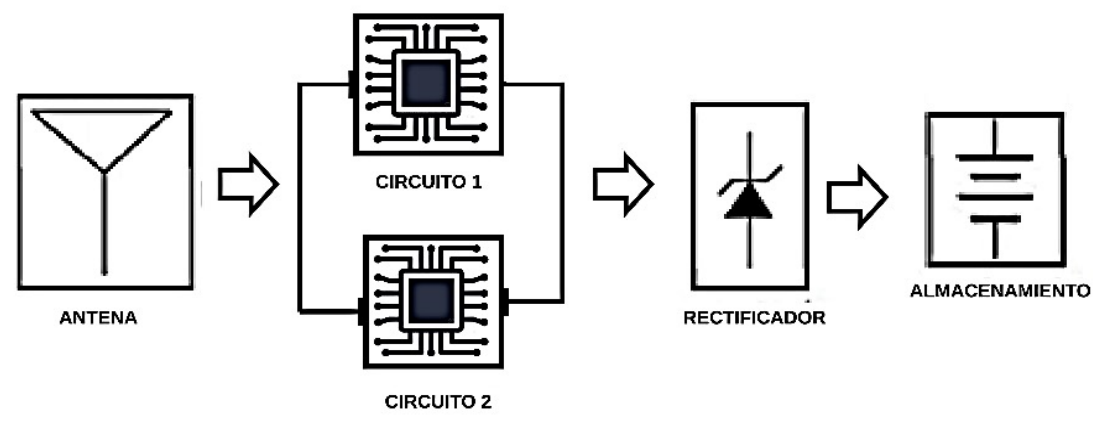

Este sistema está formado por 3 etapas que son: antena (planar dual-polarized), acoplamiento y rectificador (onda completa). La aplicación del sistema es recolección de energía inalámbrica para una gama de sensores inalámbricos y aplicaciones de red. (Chaoyun Song, 2015).

S5: Este tipo de rectena diseñada tiene un peso de $15 \mathrm{~g}$ y trabaja en las frecuencias de 915 $\mathrm{MHz}$ y $2.45 \mathrm{GHz}$ con potencia mínima de 1uW. El sistema consta de 3 etapas como se observa en la figura 4.

Las etapas constan de un arreglo de antenas Yagi-Uda para recolección de energía de radiofrecuencia. El acoplamiento es realizado al rectificador de onda completa, obteniendo energía para aplicaciones de bajo consumo de energía. (Robert Scheeler, 2014)

S6: Este tipo de sistema está conformado por 4 etapas que son utilizadas para diferentes tipos de captación de energía. El diagrama de bloques se visualiza en la figura 6.

Figura 6. Diagrama de bloques del S6

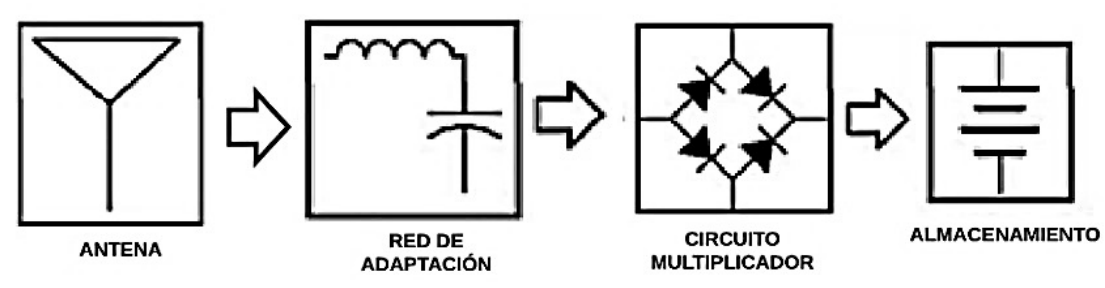

Las etapas del sistema son: antena, red de adaptación, multiplicador de voltaje y almacenamiento. La estructura interna de la etapa 1 y 2 está compuesta de elementos como: inductores, capacitores, filtros y rectificación. La frecuencia de trabajo es $3.43 \mathrm{GHz}$ y recolecta energía de radiofrecuencia, utilizando una Microstrip (antena de parche polarizada linealmente). Posteriormente, la energía es procesada por un rectificador de onda completa y un circuito multiplicador incorporado en la antena. Después, es almacenada en baterías de respaldo. (A.Rajan, 2014)

S7: Este sistema está compuesto por dos tipos de recolección de energía: 1) empleando una corriente corona y 2) señales de radiofrecuencia, obteniendo el diagrama de bloques visualizado en la figura 7 y 8. 
Figura 7. Esquema de conversión de RF a DC

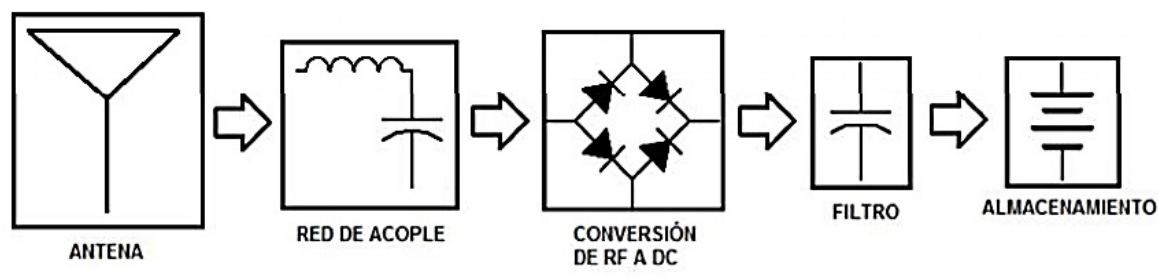

Figura 8. Esquema de conversión de corona a DC

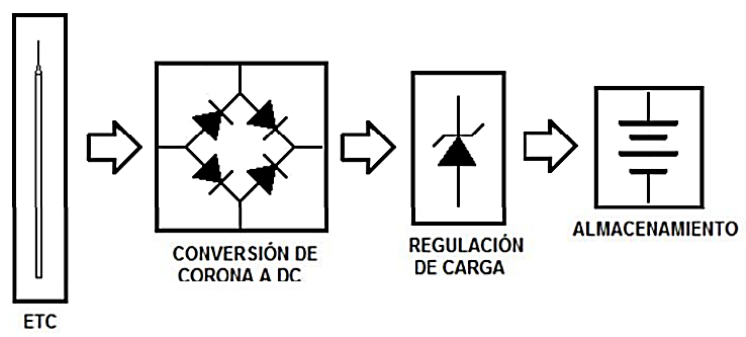

El circuito completo se obtiene de la unión de diagramas de bloques (figura 7 y 8). Adicionalmente, incorpora un diagrama de control que consta de un molino de campo y una tarjeta de adquisición dispuesta de interruptores para ambos circuitos.

La frecuencia de trabajo del sistema es de $300 \mathrm{KHz}$ a $3 \mathrm{GHz}$. El diagrama de bloques está dividido en dos partes debido a que va a recolectar dos tipos de energía, utilizando diferentes tipos de antenas que son: monopolo para energía de radiofrecuencia y electrodo tipo corona para campo eléctrico. El primer esquema tiene antena y circuito de recolección de corriente corona. Y, el segundo tiene ETC, circuito de recolección de RF, control y almacenamiento.

La estructura interna de ambos circuitos está compuesta por elementos simulares para su funcionamiento y una etapa de control. El circuito multiplicador de voltaje tiene configuración Villard y el rectificador de onda completa. La energía recolectada y procesada es almacenada en baterías de litio para su posterior aplicación en celdas foto-catalíticas para procesos de hidrolisis de bajo consumo (Guerrero, 2013).

S8: El sistema es una rectena para recolección de energía de radiofrecuencia encontrada en el ambiente. Trabaja en doble banda de $915 \mathrm{MHz}$ y $2.45 \mathrm{GHz}$. La rectena utiliza antenas dipolo plegadas de carga por ranura. El diagrama de bloques de 3 etapas del sistema se observa en la figura 4.

La etapa del rectificador se observa en la figura 9, obteniendo un voltaje de corriente continua a la salida del sistema.

Figura 9. Diagrama de bloques del diseño del rectificador para S8

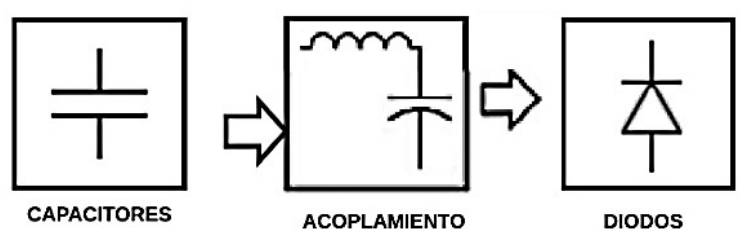


El S8 está conformado por 3 etapas que son: antena, acoplamiento y rectificador. El tipo de rectificador utilizado es de onda completa. Está formado por: capacitores, diodos y el acoplamiento a la antena. Este sistema puede ser utilizado en sensores de baja potencia y almacenamiento de energía (Kyriaki Niotaki, 2013).

S9: El sistema utiliza un arreglo de antenas Cuasi-Yagi para bandas de frecuencia de 1.8 a $2.2 \mathrm{GHz}$. Está diseñado para recolectar energía de radiofrecuencia ambiental constituido de 3 etapas. Tal como fue mostrado en la figura 4.

En la figura 10 se observa la etapa de rectificación de 4 bloques para el rectificador.

Figura 10. Diagrama de bloques del diseño del rectificador para S9

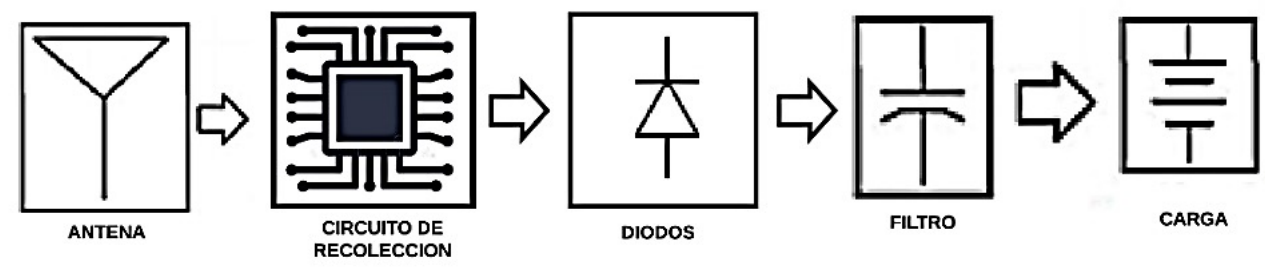

Las etapas de funcionamiento son: antena, acoplamiento y rectificador de doble banda. La estructura interna del rectificador consta de: circuitos elementales, diodos, filtros y resistencias. Su aplicación es en redes de sensores inalámbricos que dependen de sistemas autónomos (Hucheng Sun Y.-X. G., 2013).

S10: Este diseño es una rectena que trabaja a una frecuencia de $2.45 \mathrm{GHz}$ y está compuesto de 2 etapas que son: diseño de antena y rectificador mostrado en la Figura 1. El diseño del rectificador es en base al sistema de medición de la rectena realizada en el que se utilizaron dispositivos de medición (voltímetro y analizador de espectro) y un generador de señal.

La frecuencia de $2.45 \mathrm{GHz}$ es mayormente utilizada para redes inalámbricas. Las ventajas de la utilización de estas bandas son: la tolerancia al atravesar obstáculos y, según Peña (2015) menciona, es compatible con la tecnología wifi.

El tipo de antena utilizada es CPS-Microsip y un rectificador de media onda y tiene la función de recolectar energía de radiofrecuencia. El sistema se aplica como energía de suministro para muchos dispositivos eléctricos como auriculares, dispositivos médicos portátiles, sensores, entre otros (Hucheng Sun Y.-x. G., 2012).

Finalizada la revisión literaria se obtienen los parámetros de cada sistema. Un factor importante en cada sistema es su frecuencia de trabajo. Con este parámetro deberán operar todos los elementos que constituyen el sistema. Además, depende de la arquitectura utilizada que puede ser un sistema de 2 etapas hasta un sistema de 4 etapas, algunos de los sistemas no utilizan circuitos multiplicadores. Otro elemento para todos los sistemas es la etapa de recolección o antena que varía en forma y modo de recolección en cada sistema.

\section{Resultados y discusión}

Se realizó la comparativa entre sistemas y se identificaron parámetros y características como: frecuencia, arquitectura de bloques y su estructura interna, tipo de energía a recolectar, tipo de antena, circuito multiplicador, almacenamiento, rectificador y su aplicación. Como se puede observar en la Tabla 1 y 2. 
Tabla 1. Comparativa de arquitecturas de modelos de sistemas de captación de energía

\begin{tabular}{|c|c|c|c|c|c|}
\hline & S1 & S2 & S3 & S4 & S5 \\
\hline Frecuencia & $\begin{array}{l}840 \mathrm{MHz}, 1,86 \\
2,1 \text { y } 2,45 \mathrm{GHz}\end{array}$ & $1.84 \mathrm{GHz}$ & $915 \mathrm{MHz}$ & 1.8 a $2.5 \mathrm{GHz}$ & $915 \mathrm{MHz}$ y $2.45 \mathrm{GHz}$ \\
\hline Tipo de antena & $\begin{array}{l}\text { Antena } \\
\text { multibanda }\end{array}$ & $\begin{array}{l}\text { Arreglo de antenas } \\
\text { Dipolo }\end{array}$ & Monopolo espiral & $\begin{array}{l}\text { A planar } \\
\text { dual-polarized }\end{array}$ & $\begin{array}{l}\text { Arreglo de antenas } \\
\text { Yagi-uda }\end{array}$ \\
\hline $\begin{array}{c}\text { Circuito } \\
\text { multiplicador }\end{array}$ & N/A & $\begin{array}{l}\text { Configuración } \\
\text { con dos diodos } \\
\text { Schottky de bajo } \\
\text { voltaje }\end{array}$ & $\begin{array}{l}\text { Configuración } \\
\text { Villard }\end{array}$ & N/A & N/A \\
\hline Almacenamiento & N/A & Ninguno & Ninguno & Ninguno & Ninguno \\
\hline Rectificador & Onda completa & Onda completa & Onda completa & Onda completa & Onda completa \\
\hline Aplicación & $\begin{array}{l}\text { Aplicaciones } \\
\text { prácticas } \\
\text { de sensores } \\
\text { inalámbricos }\end{array}$ & $\begin{array}{l}\text { Circuitos elec- } \\
\text { trónicos de bajo } \\
\text { consumo de } \\
\text { potencia }\end{array}$ & $\begin{array}{l}\text { Circuitos de bajo } \\
\text { consumo de } \\
\text { potencia y recargar } \\
\text { baterías }\end{array}$ & $\begin{array}{l}\text { Gama de } \\
\text { sensores } \\
\text { inalámbricos } \\
\text { y aplicaciones } \\
\text { de red }\end{array}$ & $\begin{array}{l}\text { Aplicaciones de } \\
\text { bajo consumo de } \\
\text { energía }\end{array}$ \\
\hline
\end{tabular}

Tabla 2. Comparativa de arquitecturas de modelos de sistemas de captación de energía

\begin{tabular}{|c|l|l|l|l|l|}
\hline \multicolumn{1}{|c|}{ S6 } & \multicolumn{1}{|c|}{ S7 } & \multicolumn{1}{c|}{ S8 } & \multicolumn{1}{c|}{ S9 } & \multicolumn{1}{c|}{ S10 } \\
\hline Frecuencia & $3,43 \mathrm{GHz}$ & 300 kHz a 3GHz & $915 \mathrm{MHz}$ a 2.45 GHz & 1.8 a 2.2 GHz & $2,45 \mathrm{GHz}$ \\
\hline Tipo de antena & $\begin{array}{l}\text { Microstrip } \\
\text { (antena de } \\
\text { parche polarizada } \\
\text { linealmente) }\end{array}$ & $\begin{array}{l}\text { Circuito 1- electro- } \\
\text { do tipo corona } \\
\text { Circuito } 2 \\
- \text { monopolo }\end{array}$ & $\begin{array}{l}\text { Antena dipolo } \\
\text { plegada de doble } \\
\text { banda }\end{array}$ & $\begin{array}{l}\text { Arreglo de } \\
\text { antenas } \\
\text { cuasi-Yagi }\end{array}$ & CPS-Microsip \\
\hline $\begin{array}{c}\text { Circuito } \\
\text { multiplicador/ } \\
\text { doblador }\end{array}$ & $\begin{array}{l}\text { Incorporado en la } \\
\text { antena }\end{array}$ & $\begin{array}{l}\text { Configuración } \\
\text { Villard }\end{array}$ & N/A & N/A & N/A \\
\hline Almacenamiento & Batería de respaldo & Batería de litio & N/A & Ninguno & Ninguno \\
\hline Rectificador & Onda completa & Onda completa & Onda completa & Doble banda & Media onda \\
\hline Aplicación & $\begin{array}{l}\text { Generador de res- } \\
\text { paldo en sistemas } \\
\text { de energía }\end{array}$ & $\begin{array}{l}\text { Celda foto-catali- } \\
\text { tica para procesos } \\
\text { de hidrolisis de } \\
\text { baso consumo }\end{array}$ & $\begin{array}{l}\text { Sensores de baja } \\
\text { potencia, alma- } \\
\text { cenamiento de } \\
\text { energía }\end{array}$ & $\begin{array}{l}\text { Redes de senso- } \\
\text { res inalámbricos } \\
\text { que dependen } \\
\text { de sistemas } \\
\text { autónomos }\end{array}$ & $\begin{array}{l}\text { Energía de } \\
\text { suministro para } \\
\text { dispositivos } \\
\text { eléctricos }\end{array}$ \\
\hline
\end{tabular}

El producto obtenido es una APP de consulta con un menú de selección de las diferentes arquitecturas clasificadas por número de etapas 2, 3, 4 y 5. En la cual, al seleccionar una opción, se despliega una ventana de los sistemas con su respectiva descripción y diagrama de bloques. Tal como se visualiza en la Figura 11. 
Figura 11. Aplicación de consulta de sistemas de captación de energía
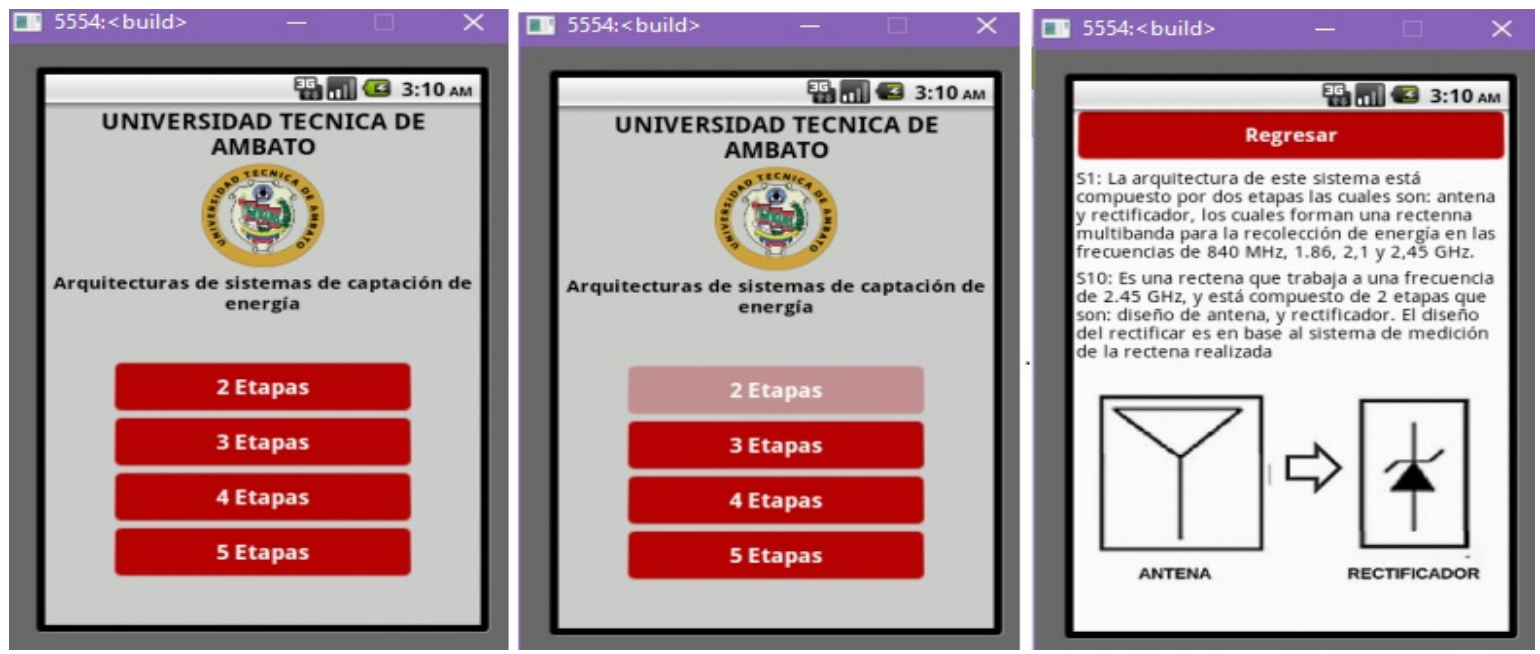

\section{Conclusiones y recomendaciones}

De la revisión de los diferentes sistemas se obtuvo la arquitectura más común y utilizada que consta de 3 etapas que son: antena, acoplamiento y rectificación. Esta arquitectura tiene como ventaja, en comparación a los demás, que su arquitectura es reducida y menos costosa. Las frecuencias de operación comprenden desde $915 \mathrm{MHz}$ hasta $3.43 \mathrm{GHz}$, siendo las frecuencias más utilizadas las que están en el rango de $2.45 \mathrm{GHz}$. Cada sistema utiliza diferentes configuraciones y tipos de antenas debido a las necesidades y requerimientos que pretenden cubrir cada uno, predominando los arreglos de antenas para controlar de mejor manera los parámetros para la captación de energía. En los circuitos multiplicadores existen diferentes configuraciones, notándose que en S2 los dos diodos de Schottky de bajo voltaje son utilizados para obtener mayor eficiencia o transmisión de la energía. La arquitectura de S6 y S7 tiene un sistema de almacenamiento con batería de respaldo y de litio, lo que permite almacenar energía para su posterior utilización. Este detalle define a estos sistemas para aplicaciones de mayor duración. En gran parte de los sistemas se utiliza la rectificación de onda completa porque la frecuencia es reducida a la mitad, permitiendo una mayor velocidad de transmisión de energía. De la revisión de los diferentes sistemas, las aplicaciones se enfocan en sistemas de bajo consumo de potencia que es útil en sistemas de transmisión inalámbrica como principios de sensores en los puntos primarios. Además, el producto obtenido es una APP de consulta con un menú de selección de las diferentes arquitecturas investigadas, lo cual es un aporte muy beneficioso para quienes deseen trabajar en esta área.

\section{Agradecimientos}

Los autores agradecen a la Universidad Técnica de Ambato y a la Dirección de Investigación y Desarrollo (DIDE) por su apoyo en la realización de esta investigación y en la ejecución del proyecto 'Sistema de Captación de Energía Electromagnética para Abastecimiento de Energía en Terminales de Internet de las Cosas (IOT) en entornos de Quinta Generación (5G)', código del proyecto: SFFISEI 04. 


\section{Referencias}

Aboueidah, H., et al. (2017). "Characterization of RF energy harvesting at $2.4 \mathrm{GHz}$ ". 24th IEEE International Conference on Electronics, Circuits and Systems (ICECS): 446-449. http://doi.org/10.1109/ IECS.2017.8292118

Abouzied, M.A., Ravichandran, K.; Sánchez-Sinencio, E. (2017). A Fully Integrated Reconfigurable Self-Startup RF Energy-Harvesting System With Storage Capability. IEEE Journal of Solid-State Circuits 52(3): 704-719. http://doi.org/10.1109/JSSC.2016.2633985

Al-Azawy, M.M.; Sari, F. (2019). Analysis of Dickson Voltage Multiplier for RF Energy Harvesting.1st Global Power, Energy and Communication Conference (GPECOM): 10-14. http://doi.org/10.1109/GPECOM.2019.8778492

Arrawatia, M.; Baghini, M.S.; Kumar, G. (2015). Differential Microstrip Antenna for RF Energy Harvesting. IEEE Transactions on Antennas and Propagation 63(4): 158-1588. http://doi.org/10.1109/ TAP.2015.2399939

Bai, X.; Ali, T.; Xu, L. (2019). A Dual-frequency Slotted CPW Antenna for 2.45/5.8 GHz RF Energy Harvesting Based on PVDF. International Applied Computational Electromagnetics Society Symposium. China (ACES): 1-2. http://doi.org/10.23919/ACES48530.2019.9060561

Cansiz, M.; Altinel, D.; Kurt, G.K. (2019). Efficiency in RF energy harvesting systems: A comprehensive review. Energy 174: 292-309. http://doi.org/10.1016/j.energy.2019.02.100

Chaudhary, S.; Johari, R.; Bhatia, R.; Gupta, K.; Bhatnagar, A. (2019). CRAloT: Concept, Review and Application(s) of loT. 4th International Conference on Internet of Things: Smart Innovation and Usages (IoT-SIU): 1-4. http://doi.org/10.1109/IoT-SIU.2019.8777467

Chouhan, S.S.; Halonen, K. (2014). Voltage multiplier circuit for UHF RF to DC conversion for RFID applications. NORCHIP: 1-4. http://doi.org/10.1109/NORCHIP.2014.700471

Chouhan, S.S.; Nurmi, M.; Halonen. K. (2016). Efficiency enhanced voltage multiplier circuit for RF energy harvesting. Microelectronics Journal 48: 95-102. http://doi.org/doi:10.1016/j.mejo.2015.11.012

Dardeer, O.M.A.; Elsadek, H.A.; Abdallah, E.A.; Elhennawy, H.M. (2019). 4x4 Circularly Polarized Antenna Array for Ambient RF Energy Harvesting. IEEE International Symposium on Antennas and Propagation and USNC-URSI Radio Science Meeting: 1175-1176. http://doi.org/10.1109/APUSNCURSINRSM.2019.8888857

Eid, A.; Constantine, J.; Tawk, Y.; Ramadan, H.; Abdalah, M.; Elhaj, R.; Awad, R.; Kasbah, B. (2017). An efficient RF energy harvesting system. 11th EUCAP: 896-899. http://doi.org/10.23919/EuCAP.2017. 7928573

Elsheakh, D.N. (2019). Broadband Dual Linear Polarized (DLP) Antenna Array for Energy Harvesting System. IEEE International Symposium on Antennas and Propagation and USNC-URSI Radio Science Meeting: 1353-1354. http://doi.org/10.1109/APUSNCURSINRSM.2019.8888786

Garg, N.; Garg, R. (2017). Energy harvesting in loT devices: A survey. International Conference on Intelligent Sustainable Systems (ICISS): 127-131. http://doi.org/10.1109/ISS1.2017.8389371

Gomez D.L. (2020). Caracterización experimental de una pmu (Power Management Unit) para aplicaciones de Energy Harvesting (EH) (Tesis de grado) Universitat Politècnica de Catalunya. https:// upcommons.upc.edu/handle/2117/329335

González, L.; Osiris, S.; Laguía, D.; Gesto, E.; Hallar, K. (2020). Internet del futuro- estudio de tecnologías IoT. ICT-UNPA 12(3): 105-137. http://doi.org/10.22305/ict-unpa.v12.n3.744

Gosset, G.; Flandre, D. (2011). Fully-Automated and Portable Design Methodology for Optimal Sizing of Energy-Efficient CMOS Voltage Rectifiers. IEEE Journal on Emerging and Selected Topics in Circuits and Systems 1(2): 141-149. http://doi.org/10.1109/JETCAS.2011.2158357

Gudan, K.; Chemishkian, S.; Hull, J.J.; Thomas, S.J.; Ensworth, J.; Reynolds, M.S. (2014). A 2.4GHz ambient RF energy harvesting system with -20dBm minimum input power and NiMH battery storage. IEEE RFID-TA: 7-12. http://doi.org/10.1109/RFID-TA.2014.6934191 
Guerrero, C. A. (2016). Sistema de recolección de energía (Energy Harvesting) que emplea la corriente corona y señales de alta frecuencia (tesis de Maestría). Universidad Nacional de Colombia, Colombia. https://repositorio.unal.edu.co/handle/unal/59817

Gupta, A.K.; Johari, R. (2019). IOT based Electrical Device Surveillance and Control System. 4th International Conference on Internet of Things: Smart Innovation and Usages (IOT-SIU).1-5. http://doi. org/10.1109/IOT-SIU.2019.8777342

Haddad, P.; Gosset, G.; Raskin, J.; Flandre, D. (2014). Efficient ultra low power rectification at $13.56 \mathrm{MHz}$ for a $10 \mu \mathrm{A}$ load current. SOI-3D-Subthreshold Microelectronics Technology Unified Conference (S3S): 1-2. http://doi.org/10.1109/S3S.2014.7028220

Hamza, T.; Adel, B.; Reda, B.M. (2020). A Low-Cost Elliptical Triple-Band Antenna for RF Energy Harvesting Applications. 5th International Conference on Renewable Energies for Developing Countries (REDEC): 1-3. http://doi.org/10.1109/REDEC49234.2020.9163859

HarshaVardhan, B.; Jagadeesh, R.; Prasad, C.; Natarajamani, S. (2019). Design of Rectifier at ISM Band for RF Energy Harvesting of Low Powers. International Conference on Communication and Signal Processing (ICCSP): 0282-0285. http://doi.org/10.1109/ICCSP.2019.8697979

Jabbar, H.; Song, Y.S.; Jeong, T.T. (2010). RF energy harvesting system and circuits for charging of mobile devices. IEEE Transactions on Consumer Electronics 56(1): 247-253. http://doi.org/10.1109/ TCE.2010.5439152

Kanaya, H. (2014) Multi-band miniaturized slot antenna with multi-band impedance matching circuit. Proceedings of 2014 3rd Asia-Pacific Conference on Antennas and Propagation: 551-554. http:// doi.org/10.1109/APCAP.2014.6992552

Khan, M.S. y Deng, H. (2016). Design and implementation of a highly efficient UHF energy harvesting antenna. IEEE International Symposium on Antennas and Propagation (APSURSI): 611-612. http:// doi.org/10.1109/APS.2016.7696014

Kundu, A.K.; Kumar-Varshney, S. (2018). Design of a Rectenna for Ambient Energy Harvesting. IEEE MTT-S International Microwave and RF Conference (IMaRC): 1-3. http://doi.org/10.1109/ IMaRC.2018.8877211

Kurvey, M.; Kunte, A. (2018). Design and optimization of stepped rectangular antenna for RF energy harvesting. International Conference on Communication information and Computing Technology (ICCICT): 1-4. http://doi.org/10.1109/ICCICT.2018.8325885

Larrocha, B. P. (2018). Double Smart Energy Harvesting System for self-powered Industrial IoT (Tesis doctoral). Universidad del País Vasco. https://addi.ehu.es/handle/10810/32071

Lauder, D.; Sun, Y. (2020). Design Considerations of Antennas and Adaptive Impedance Matching Networks for RF Energy Harvesting. European Conference on Circuit Theory and Design (ECCTD): 1-4. http://doi.org/10.1109/ECCTD49232.2020.9218310

Lee, D.; Kim, T.; Kim, S.; Byun, K.; Kwon, K. (2018). A CMOS Rectifier with 72.3\% RF-to-DC Conversion Efficiency Employing Tunable Impedance Matching Network for Ambient RF Energy Harvesting. International SoC Design Conference (ISOCC): 259-260. http://doi.org/10.1109/ISOCC.2018.8649983

Mahendra, A.; Adhiyoga, Y.G.; Broto, W.; Saputra, A.; Pratomo, V.A. (2019). Numerical Design of Quad-Band Antenna for UWB RF Signal Energy Harvesting on IoT Application. IEEE Conference on Sustainable Utilization and Development in Engineering and Technologies (CSUDET): 281-283. http:// doi.org/10.1109/CSUDET47057.2019.9214583

Maher, R.; Tammam, E.; Galal, A.I.; Hamed, H.F. (2016). Design of a broadband planar antenna for RF energy harvesting. International Conference on Electrical, Electronics, and Optimization Techniques (ICEEOT): 1808-1810. http://doi.org/10.1109 / ICEEOT.2016.7754999

Martínez, J.; Medina, A.S.; Bonilla, C.A.; Villegas, J.M.; y Aldaz, J.C. (2020). Radio Frequency Energy Harvesting System Making Use of $180^{\circ}$ Hybrid Couplers and Multiple Antennas to Improve the DC Output Voltage. IEEE Latin America Transactions 18(3): 604-612. http://doi.org/10.1109/ TLA.2020.9082733 
Mindan, B.; Hong, L. (2010). The Analysis of Impedance Matching Problem in RF Circuit Design. International Forum on Information Technology and Applications: 350-353. http://doi.org/10.1109/ IFITA.2010.55

Moghaddam, N.A.; Maleki, A.; Shirichian, M.; Panah, N.S. (2017). RF energy harvesting system and circuits for charging of wireless devices using spectrum sensing. 24th IEEE International Conference on Electronics, Circuits and Systems (ICECS): 431-436. http://doi.org/10.1109/ ICECS.2017.8292044

Morales, H.; Dunleavy, L. Skidmore, S. (2012). Ultra-broadband characterization of Schottky diodes. 79th ARFTG Microwave Measurement Conference: 1-4. http://doi.org/10.1109/ ARFTG79.2012.6291198

Mouapi, A.; Hakem, N.; Kandil, N. (2018). High Efficiency Voltage Doubler Rectifier Design for Harvesting ambient RF Energy. IEEE International Symposium on Antennas and Propagation \& USNC/URSI National Radio Science Meeting: 2515-2516. http://doi.org/10.1109/APUSNCURSINRSM.2018.8609029

Nalini, M.; Kumar, J.V.N.; Kumar, R.M.; Vignesh, M. (2017). Energy harvesting and management from ambient RF radiation. International Conference on Innovations in Green Energy and Healthcare Technologies (IGEHT): http://doi.org/10.1109/IGEHT.2017.8094073

Niotaki, K.; Kim, S.; Jeong, S.; Collado, A.; Georgiadis, A.; Tentzeris, M.M. (2013). A Compact Dual-Band Rectenna Using Slot-Loaded Dual Band Folded Dipole Antenna. IEEE Antennas and Wireless Propagation Letters 12: 1634-1637. http://doi.org/10.1109/LAWP.2013.2294200

Nintanavongsa, P.; Muncuk, U.; Lewis D.R.; Chowdhury, K.R. (2012). Design Optimization and Implementation for RF Energy Harvesting Circuits. IEEE Journal on Emerging and Selected Topics in Circuits and Systems 2(1): 24-33. http://doi.org/10.1109/JETCAS.2012.2187106

Park, D.; Bang, H.; Pyo, C.S.; Kang, S. (2014). Semantic open loT service platform technology. IEEE World Forum on Internet of Things (WF-IOT): 85-88. http://doi.org/10.1109/WF-IoT.2014.6803125

Peña, R. P. (2015). Planteamiento de conexión alternativa a red móvil para acceso de conectividad a datos basado en el estándar 802.11 (Tésis de maestria) Pontificia Universidad Católica del Ecuador, Ecuador. http://repositorio.puce.edu.ec/handle/22000/12497

Piñuela, M.; Mitcheson, P.D.; Lucyszyn, S. (2013). Ambient RF Energy Harvesting in Urban and Semi-Urban Environments. IEEE-TMTT 61(7): 2715-2726. http://doi.org/10.1109/TMTT.2013.2262687

Polaiah, G.; Krishnamoorthy, K.; Kulkarni, M. (2019). A Compact Dual-Band Rectenna for RF Energy Harvesting. IEEE Indian Conference on Antennas and Propogation (InCAP): 1-4. http://doi.org/10.1109/ InCAP47789.2019.9134462

Ramesh, G.P.; Rajan, A. (2014). Microstrip antenna designs for RF energy harvesting. International Conference on Communication and Signal Processing: 1653-1657. http://doi.org/10.1109/ ICCSP.2014.6950129

Salih, A.A.; Sharawi, M.S. (2015). A miniaturized dual-band meander line antenna for RF energy harvesting applications. IEEE Jordan Conference on Applied Electrical Engineering and Computing Technologies (AEECT): 1-4. http://doi.org/10.1109/AEECT.2015.7360530

Saxena, A; Shinghal, K.; Misra, R.; Agarwal, A. (2019). Automated Enhanced Learning System using IOT. 4th International Conference on Internet of Things: Smart Innovation and Usages (IoT-SIU): http:// doi.org/10.1109/loT-SIU.2019.8777711

Scheeler, R.; Korhummel, S.; Popovic, Z. (2014). A Dual-Frequency Ultralow-Power Efficient 0.5-g Rectenna. IEEE Microwave Magazine 15,(1): 109-114. http://doi.org/10.1109/MMM.2013.2288836

Sedeek, A.; Tammam, E.; Hasaneen, E.S. (2018). High Efficiency 2.45 GHz Low Power Hybrid Junction Rectifier for RF Energy Harvesting. International Japan-Africa Conference on Electronics, Communications and Computations (JAC-ECC):147-150. http://doi.org/10.1109/JEC-ECC.2018.8679546 
Shen, S.; Chiu, C.; Murch, R.D. (2017). A Dual-Port Triple-Band L-Probe Microstrip Patch Rectenna for Ambient RF Energy Harvesting. IEEE Antennas and Wireless Propagation Letters 16: 3071-3074. http://doi.org/10.1109/LAWP.2017.2761397

Song, C.; Huang, Y.; Zhou, J.; Zhang, J.; Yuan, S.; Carter, P. (2015). A High-Efficiency Broadband Rectenna for Ambient Wireless Energy Harvesting. IEEE Transactions on Antennas and Propagation 63(8): 3486-3495. http://doi.org/10.1109/TAP.2015.2431719

Sun, H.; Guo, Y.; He, M.; Zhong, Z. (2012). Design of a High-Efficiency 2.45-GHz Rectenna for Low-InputPower Energy Harvesting. IEEE Antennas and Wireless Propagation Letters 11: 929-932. http:// doi.org/10.1109/LAWP.2012.2212232

Sun, H.; Guo, Y.; He, M.; Zhong, Z. (2013). A Dual-Band Rectenna Using Broadband Yagi Antenna Array for Ambient RF Power Harvesting. IEEE Antennas and Wireless Propagation Letters 2: 918-921. http://doi.org/10.1109/LAWP.2013.2272873

Tony, A; Hiryanto, L. (2019). A review on energy harvesting and storage for rechargeable wireless sensor networks. IOP Conference Series: Materials Science and Engineering. 508(1): 651-654. http:// doi.org/10.1088/1757-899X/508/1/012120

Torrealba, R.; Santiesteban, V.C.; Ambrosio, R.; Gomez, L.; Flores, E. (2016). Sistema de cosechamiento de energía con radio frecuencia. SOMI XXXI: 1-6

Tran, L-G.; Cha, H-K.; Park, W-T. (2017). RF power harvesting: a review on designing methodologies and applications. Micro and Nano Systems Letters, 5(14): 1-16. http://doi.org/10.1186/s40486-0170051-0

Vishwakarma, S.K.; Upadhyaya, P.; Kumari, B.; Mishra, A.K. (2019). Smart Energy Efficient Home Automation System Using loT. 4th International Conference on Internet of Things: Smart Innovation and Usages (IOT-SIU): 1-4. http://doi.org/10.1109/IoT-SIU.2019.8777607

Vu, H. S., et al., (2020). Multiband Ambient RF Energy Harvesting for Autonomous IoT Devices. IEEE Microwave and Wireless Components Letters (30): 1189-1192. http://doi.org/10.1109/ LMWC.2020.3029869

Wen, J.; Xie, D.; Liu, X.; Guo, H.; Liu, C.; Yang, X. (2016). Antena en forma de collar de banda ancha para recolección de energía de RF. Simposio internacional de Asia y el Pacífico sobre compatibilidad electromagnética (APEMC): 253-255. http://doi.org/10.1109 / APEMC.2016.7523025

Yoshikawa, T. (2019). The Efficiency Estimation Method for Harvesting Energy Charged into Capacitor. IEEE Third International Conference on DC Microgrids (ICDCM): 1-5. http://doi.org/10.1109/ ICDCM45535.2019.9232851

Zainuddin, N.A., et al. (2013). Design of wideband antenna for RF energy harvesting system. 3rd International Conference on Instrumentation, Communications, Information Technology and Biomedical Engineering (ICICI-BME): 162-166. http://doi.org/10.1109/ICICI-BME.2013.6698485. 